\title{
AVALIAÇÃO DOS PACIENTES COM VARIZES ESOFÁGICAS HEMORRÁGICAS SUBMETIDOS À ESCLEROTERAPIA ENDOSCÓPICA
}

\author{
RENER GUERRA BALAN" \\ MILTON SHIGUEKI NAGAOKA: \\ INÉS OGAI NAKAMOTO' \\ ROSE MEIRE ALBUQUERQUE PONTES ${ }^{2}$ \\ EVELIN MASSAE OGATTA MURAGUCHI \\ FAISSAL JOSÉ MUARREK² \\ PEDROHUMBERTO PERINLEITE"
}

\begin{abstract}
BALAN, Rener Guerra; NAGAOKA, Milton Shigueki Nagaoka; NAKAMOTO, Inês Ogai; PONTES, Rose Meire Albuquerque; MURAGUCHI, Evelin Massae Ogata; MUARREK, Faissal José; LEITE, Pedro Humberto Perin. Avaliação dos pacientes com varizes esofágicas hemorágicas submetidos à escleroterapia endoscópica.

Semina: Ci. Biol./Saúde. Londrina, v. 16, n. 2, p. 292-294, jun 1995.
\end{abstract}

RESUMO: A hemorragia alta por varizes de esôfago é uma das complicaçōes mais graves de pacientes com hipertensão portal e a escleroterapia endoscopica tem sido utilizada para o iratamento e profilaxia do ressangramento.

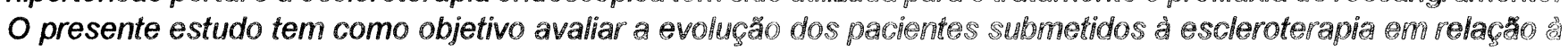
presença de ressangramento e as complicaçós, num periodo de dois anos. DOS 31 pacientes quse

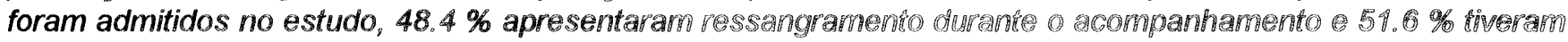
complicaçðes, sendo a úlcera esofágica mais frequiente (56.3\%).

PALAVRAS CHAVE: varizes esofágicas, escleroterapia endoscópica, ülcera esofágica.

\section{INTRODUÇÃO}

A probabilidade de um paciente ressangrar após ( ) primeiro sangramento por varizes esofágicas è de aproximadamente $70 \%$ nas seis primeiras semanes seguintes, diminuindo essa incidência gradualmente. Os métodos conhecidos para a prevençăo de ressangramento e os procedimentos cirúrgicos visam reduzir a hipertensão portal. Estes últimos constituem arma de maior efetividade mas limitados pela possibilidade de encefalopatia hepática. A escleroterapia endoscópica e o uso de propranolol apresentam resultados controversos, como sugere RIKKERS (1988).

Este estudo tem por objetivo conhecer a população de pacientes que frequenta o Programa de Esclerose Endoscópica Ambulatorial do Hospital Universitário de Londrina, avaliando o ressangramento e as possíveis complicações decorrentes da escleroterapia endoscópica em 31 pacientes submetidos 20 procedimento.

\section{PACIENTES E MÉTODO}

No período de 1984 a 1994, no Hospital Universitário de Londrina, oitenta e oito pacientes entraram no programa de esclerose endoscópica de varizes, por terem apresentado hemorragia digestiva alta por varizes de esôfago. Desse total, trinta e um pacientes foram admitidos no estudo, por estarem freqüentando regulamente o programa, com última revisão hå . máximo oois anos. Assim, cinqüenta e cinco pacientes foram excluidos por estarem sem revisão nos uittimos dois anos (abandono,

por parte do paciente, do tratamento e perda de contato).

A hemorragia digestiva alta por varizes de esoffago foi definida clinicamente pela presença de hematêmese ou melena : exame endoscópico, que foi realizado nas primeiras 24. horas após o sangramento.

(1) diagnóstico etiológico da doença de base fo̊ estabelecido através do quadro clínico, avaliaçăo do função hepática, uttra-sonografia e, quando possivel. biópsia hepática guiada por laparoscopia.

Os pacientes com diagnóstico de cirrose hepática foram classificados conforme o grau de insuficiência hepática segundo os critérios de Child-Pugh.

Para a escleroterapia utilizou-se solução de oleato de monoetanolamina a $2.5 \%$, com injeção de $2.3 \mathrm{ml}$ dentro do cordão varicoso, num total de 20 a $40 \mathrm{ml}$ por sessão. A primeira sessão era realizada em no máximo 2. horas após o inicio do sangramento.

Se houvesse persistência de sangramento, era colocado (1) balão de Sengstaken-Blakemore por 24-36 horas e mova esclerose após sua retirada.

A seguñda sessão era efetivada após uma semana da primeira; a terceira após 15 dias e as subsegüentes mensalmente até sua redução para

1 - Residente de Gastroenterologia Clínica

2. Docente do Departamento de Clínica Médica da Disciplina de Gastroenterologia Clinica - Centro de Ciências da Saúde - Universidade Estadual de Londrina - Caixa Postal 6001 - CEP 86051.970 
tamanho minimo; depois bimensalmente até desaparecimento completo das varizes. Após seu desaparecinento, realizavase controle endoscópico anual.

Na primeira endoscopia, as varizes eram classificadas segundo a Sociedade Japonesa de Endoscopia:

grosso calibre - grandes cordoes, espiraladas ultrapssando $1 / 3$ da luz esofágica.

médio calibre - quando tontuosas e ocupando menos de $1 / 3$ \&

pequeno calibre - quando apresentavam cordőes lineares năo lonuosos.

Utilizou-se o Método Exato de Ficher para testar a significância estatistica oos achados, com nivel de significância de cifrs de 0.05, devido ao numero pequeno da amostra.

\section{RESULTAOOS}

Orservoun, oue do doral de 31 parientes estudados, 50.0\% eram de Lomonna, Sendo os demais de cidades vizinhas. Minte o três (7. 2\%) pacientes eram do sexo mascuhno (2) (25.8\%) do sexo feminino. A

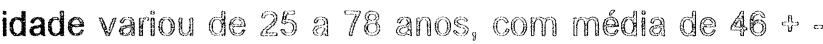
12.65 anos.

Quinze (48.4\%) pacientes estavam antre 1 is 3 anos no programa de esclerose, foratin necessainios no minimo 5 sessões de escleroraraia para desaparecimento das varizes para $32.3 \%$ dos pacientes. Os demais requereram mumero maior.

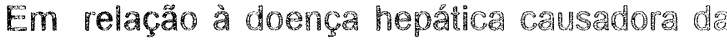

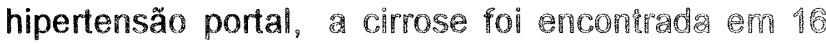
(51.6\%) pacientes: 12 por ingestäo alcoolica pơs. hepatite. Observou-se que, desses pacientes cirróicos, $2(11.8 \%)$ eram child A, 10 (53.8\%) eram Child B 5 (29.4\%) eram Child C. A esquistossomose foi obsenada em 12 pacientes $(38.7 \%)$; esquistossomose c cirrose em $1(3.2 \%)$ com patologia nă definida om 2 $(6.5 \%)$.

Considerando-se o calibre das varizes obsevado durante a primeira endoscopia, $27(87.1 \%)$ dos pacientes apresentavam grosso calibre, $4(12.7 \%)$ varizes de médio catibre nãa se obsenou a oconência de pequeno calibre.

Dos 3 1 pacientes estudados, 15 (48.4\%) apresenciaram pelo menos 1 episódio de ressangramento Has $\mathrm{vanizs}$. Desses, $14(93.3 \%)$ tinham varizes de grosso callore a $10.7 \%$ varizes de médio calibre.

O messangramento na pôde ser associado

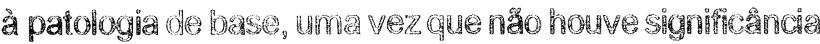

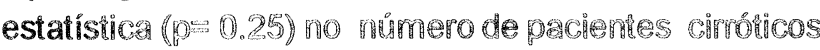
e esquishossomónicos que ressangraram.

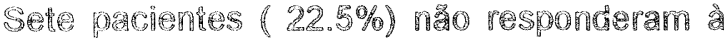

terapêtuico endoscópica, sendo submetidos iniervengo cinirgica (esplenectomia com ligadur gastro-esorågica d dervagyo sizigo portal).

Complicaçes devido ascleroterapia ocorreram em $13(4.9 \%)$ pachencs, sendo em $3(1.7 \%)$ estenose esofágica, 1 (6.3\%) paciente com sangramento e 9 (56.3\%) com ülcera esofágica. As conplicacoes, como um todo, ocorreram independente do callibre diss varizes na primeira endoscopia $(0=1.00)$, entoora lodos os pacientes com incera sofágica rivessen varizes de grosso calibre.

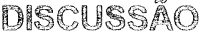

TERES \& BOSCH (1993) estudand pardentes

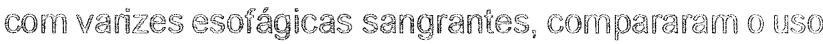

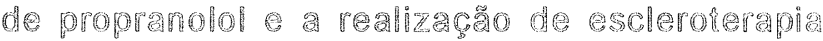

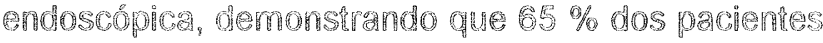
do grepo do propranolol ressangram contra As \% da ascheroterais. conchniram oruesie procedmento ara mais aricaz oue o uso do propranolol. No presente osiudo, d.6.9\% dos paciencs ressangram, o gue Se assemeng a seu achado. Cono mo homy

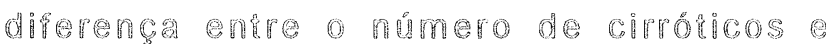

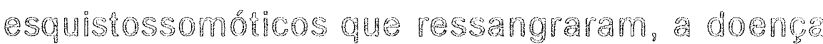
de base no hoi deteminante park o indice do ressamgramento.

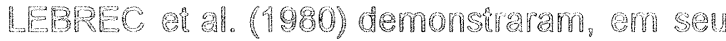
sstudo com paciences cirromicos, oue o rallibre das

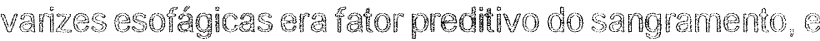
os resultados ofotidos neste frabalho demonstram gue

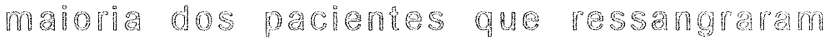
apresentavm à prineira andoscopis varizes ob grosso callore.

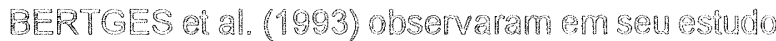

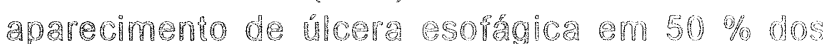
pacientes.

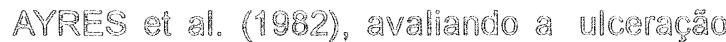

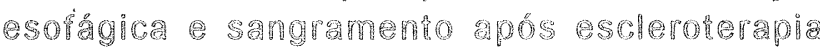

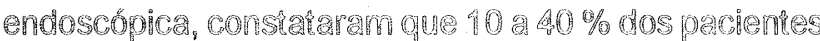

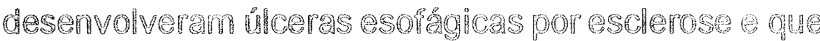

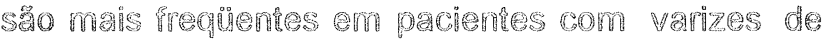
grosso calibr. O presente esudo mostron aresenge de conphicacoes an $1.9 \%$ olos parentes, sendo a mais

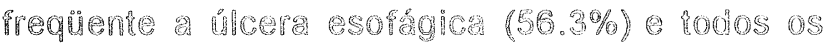

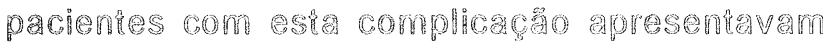

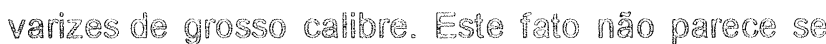

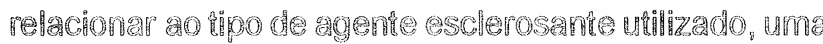
Voz ong on GREORY (1987) Dncontrar mindicos semelhantes

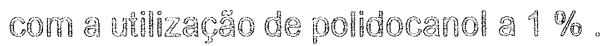

\section{CONCHUSORE}

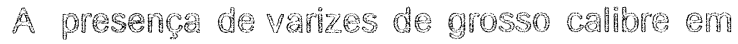


pacientes portadores de doença hepática predispõe ao sangramento por rotura das varizes, e estes pacientes são os que estão mais sujeitos às complicações, mais freqüentemente se observando a formação de úlcera esofágica.

Em cerca de $51 \%$ dos casos, o programa de escleroterapia endoscópica foi efetiva em manter 0 paciente sem sangramento pelas varizes.

BALAN, Rener Guerra; NAGAOKA, Milton Shigueki Nagaoka; NAKAMOTO, Inês Ogai; PONTES, Rose Meire Albuquerque; MURAGUCHI, Evelin Massae Ogata; MUARREK, Faissal José; LEITE, Pedro Humberto Perin. Evaluation of patients with hemorragic varices submetid to endoscopic sclerotherapy. Semina: Ci. Biol./Saúde, Londrina, v. 16, n. 2, p. 292-294, Jun. 1995.

ABSTRACT: The high digestive bleeding by esophageal varices is one of the most dramatic complications of patients with portal hypertension and the endoscopic sclerotherapy has been used on the treatment and as 50 a prevention from rebleeding. The present study has as objective to evaluate the evolution of patients submited fo sclerotherapy related to the presense of rebleeding and method complications, on a minimun period of two years. Thirty one patients took part in the study, 48.4\% of which had rebleeding during the follow 190 and $59.6 \%$ complications due to sclerosis; the esophagic ulceration was the most frequent complication (56.3\%).

KEY WORDS: esophageal varices, endoscopic sclerotherapy, Sophagic ulceration.

\section{REFERENNGIAS BIBLIOGRÁFICAS}

AYRES, S.J; GOFF, J.S。; WARREN, G.Ho; SCHAFER, J.W. Esophageal ulceration and bleeding after flexible fiberoptic esophagealvein sclerosis. Gastroenterology. v. 83, p. 1136, 1982

FLEIG, W.E.; STANGE, E.F. Esophageal varices: current therapy in 1989. Endoscopy, v.21, p.89-96, 1989 .

GREGORY, P. et al. Prophilactic sclerotherapy esophageal varices in alcoholic liver disease: resultats of a VA cooperative randomized trial. Gastroenterology, v. 92, p. 1414, 1987.

LEBREC, D. et al. Portal hipertension, size of esophageal varices and risk of gastrointestinal bleeding im

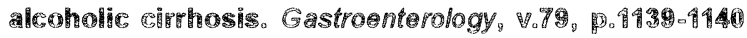
1980.

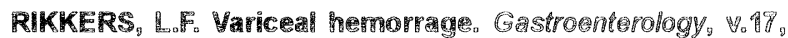
. 289.3029 .998$.

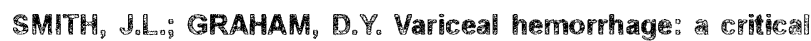

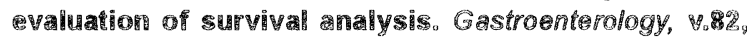
19. $368,1982$.

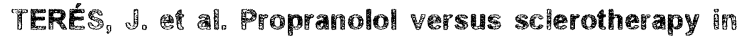
preventirg variceal rebleeding:

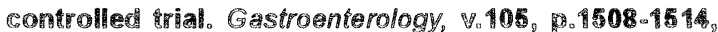
1998. 